\title{
DÜBLIN
}

Technological University Dublin

ARROW@TU Dublin

2010-9

\section{Investigating Ultrasonic Positioning on Mobile Phones}

\author{
Viacheslav Filonenko \\ Technological University Dublin, viacheslav.filonenko@tudublin.ie \\ Charlie Cullen \\ Technological University Dublin, charlie.cullen@tudublin.ie \\ James Carswell \\ Technological University Dublin, james.carswell@tudublin.ie
}

Follow this and additional works at: https://arrow.tudublin.ie/dmccon

Part of the Databases and Information Systems Commons

\section{Recommended Citation}

Filonenko, V., Cullen, C. \& Carswell, J. (2010) Investigating Ultrasonic Positioning on Mobile Phones. International Conference on Indoor Positioning and Indoor Navigation (IPIN), Zürich, Switzerland, September.

This Conference Paper is brought to you for free and open access by the Digital Media Centre at ARROW@TU Dublin. It has been accepted for inclusion in Conference papers by an authorized administrator of ARROW@TU Dublin. For more information, please contact arrow.admin@tudublin.ie, aisling.coyne@tudublin.ie, gerard.connolly@tudublin.ie.

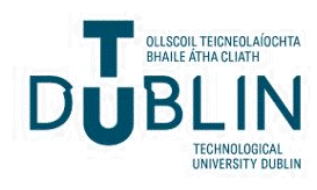


Dublin Institute of Technology

ARROW@DIT

Articles

Digital Media Centre

2010-09-01

\section{Investigating Ultrasonic Positioning on Mobile Phones}

Viacheslav Filonenko

Dublin Institute of Technology, viacheslav.filonenko@dit.ie

Charlie Cullen

Dublin Institute of Technology, charlie.cullen@dit.ie

James Carswell

Dublin Institute of Technology, jcarswell@dit.ie

\section{Recommended Citation}

Filonenko, V., Cullen, C., Carswell, J.: Investigating Ultrasonic Positioning on Mobile Phones. International Conference on Indoor Positioning and Indoor Navigation (IPIN), Zürich, Switzerland; 2010 IEEE Xplore, September, 2010.

This Conference Paper is brought to you for free and open access by the Digital Media Centre at ARROW@DIT. It has been accepted for inclusion in Articles by an authorized administrator of ARROW@DIT. For more information, please contact yvonne.desmond@dit.ie, arrow.admin@dit.ie. 


\title{
Investigating Ultrasonic Positioning on Mobile Phones
}

\author{
Viacheslav Filonenko, Charlie Cullen and James Carswell \\ Digital Media Centre, Dublin Institute of Technology, Ireland \\ \{viacheslav.filonenko, charlie.cullen, jcarswell\}@dit.ie
}

\begin{abstract}
In this paper we evaluate the innate ability of mobile phone speakers to produce ultrasound and the possible uses of this ability for accurate indoor positioning. The frequencies in question are a range between 20 and $22 \mathrm{KHz}$, which is high enough to be inaudible but low enough to be generated by standard sound hardware. A range of tones is generated at different volume settings on several popular modern mobile phones with the aim of finding points of failure. Our results indicate that it is possible to generate the given range of frequencies without significant distortions, provided the signal volume is not excessively high. This is preceded by the discussion of why such ability on off-the-shelf mobile devices is important for Location Based Services (LBS) applications research. Specifically, this ability could be used for indoor sound trilateration positioning. Such an approach is uniquely characterized by the high accuracy inherent to sound trilateration, with little computational burden on the mobile device, and no specialized hardware or audible noise. Combined with a fast internet connection and the sensors present in modern smartphones, such as accelerometers and magnetometers, our approach makes mobile phones a suitable platform for indoor LBS positioning.
\end{abstract}

Keywords - Ultrasound; Indoor Positioning; Mobile Devices

\section{INTRODUCTION}

Currently outdoor Location Based Services (LBS) have the advantage of reliable positioning via GPS (also Wi-Fi and GSM) and a defined business model for the delivery of content to the user. This has led outdoor LBS to greatly expand in recent years, though indoor locationing technologies and methods have yet to fully mature on mobile devices. In the current state of the art in indoor LBS, merging accurate indoor positioning and context-sensitive services is still an outstanding problem. Existing systems such as employee tracking [1] using RFID/Wi-Fi tags or badges are relatively cheap to implement, but no development path for mobile device RFID currently exists in Europe. For context-sensitive services, such as a virtual tour guide, factors such as device cost, functionality and service provision are still stumbling blocks to effective implementation of solutions. A frequent example would require the user to point a device at a tag or enter an exhibit's number manually. Such approaches are time consuming, complex and require user focus (thus distracting them from the exhibits). In addition, inability to provide effective user navigation (e.g. how to find an exit) and lack of rich media multimodal interfaces has led to a disparity between device capabilities (where media delivery is a de facto standard) and quality user focused services.
Currently there are no examples of fully-functional indoor LBS for mobile phones, but theoretically they could perform a number of functions:

- Make evacuation procedure more intuitive and efficient by showing directions along the shortest path [2]. In this example it is important for the system to know $100 \%$ of the time where the user is so that he does not have a reason to panic if he suddenly realises he got lost.

- Improve navigation in shopping malls. There is already a company that collects and maintains maps of shopping malls [3]. Normally when working with an unfamiliar map it takes a significant amount of time to figure out current position and direction unless the map is stationary and the position is already marked. This makes portable maps less useful. Using indoor positioning it is possible to take better advantage of such data. Showing the current position on an interactive map would already be a significant improvement and giving instructions how to get to a particular shop would make navigation even easier.

- Given better precision it may be possible to direct the user to a particular shelf in a shop. Bearing that in mind it is possible to design a program where the user has been populating a list of things he needs to buy on his mobile phone since he last went shopping. When he enters a shop the most optimal route to collect the goods is generated and the user is instructed where to go next.

- A library catalogue combined with a navigation system that directs the user to the shelf with the book he requested.

- A museum virtual tour guide. Systems currently used in museums provide unsophisticated functionality which is very often limited to pointing at a tag or manually entering a number in order to hear a recording. A system with true indoor positioning based on a mobile phone can be used by pointing at the actual exhibit and not at a tag via directional querying. Depending on the arrangement and size of exhibits, directional querying may require very high spatial and directional accuracy. A smartphone can deliver a variety of content including audio, video, text, images or a combination of them such as a webpage. Once again because the system is continuously aware of user's location it is possible to guide the user to an exhibit he wants to see, to the exit or any other facility.

- Use in a company to track employees. Systems currently used for this purpose use Wi-Fi or RFID tags. The main problem with using tags is that while the person controlling the system knows where everyone is, an average user has no benefit from this system. A smartphone version however can offer any employee to find any other employee regardless if he is right now at his 
desk or not. Depending on the type of work this ability may turn out to be extremely valuable. Also it is not unusual for companies to issue smartphones such as Blackberries to every employee, so it is very likely that everyone is already carrying necessary hardware.

Section 2 of this paper discusses related work. Section 3 discusses our methodology and Section 4 presents results of our experiments. Finally Section 5 concludes the paper and presents directions of future work.

\section{RELATED WORK}

\section{A. Indoor Positioning}

Positioning on mobile phones is not limited to GPS. Other components commonly found in mobile phones can also be used to determine position. Methods that use propagation of Radio Frequency (RF) signals are prevalent in this field, with the exception of computer vision. In computer vision SLAM appears to be the most promising, considered by many a technology still in its infancy [4]. Computer vision, although often very accurate, is characterized by high computational load, complicated procedures of recovery from tracking failures and susceptibility to camera shake and motion blur. These problems are addressed in the studies done by Williams et al. [5] and Wagner et al. [6]. Another difficulty associated with computer vision is that the user is supposed to be looking through the screen when using the device.

Every modern smartphone at least has GSM, Wi-Fi and Bluetooth modules. Five meter accuracy, one of the best results for indoor GSM positioning, was displayed by Otsason et al. with the help of wide signal-strength fingerprinting [7]. Unfortunately wide signal-strength fingerprinting is impossible on many modern phones due to OS restrictions. Other GSM positioning methods are generally impractical for indoor use due to poor accuracy. Wi-Fi positioning in average shows twice as better accuracy than GSM. A method proposed by Ferris et al. where Gaussian processes are used to mathematically predict signal strength in areas outside the exact spots where fingerprints were taken appears to be promising [8]. The best accuracy among commercial solutions was shown by Ekahau: 1-3 meters [1]. Because of the ability to leverage hardware already present in office areas Wi-Fi is a good choice for positioning, but it will become even better when client-toclient connections are possible with Wi-Fi Direct, which is due to appear in 2010 [9]. Bluetooth has the shortest range among the three technologies. There are two major problems that make Bluetooth positioning particularly difficult. First of all it is designed to adjust signal strength when signal becomes too strong or too weak. Disabling this feeback loop is discussed by Zhou et al. [10]. Another problem is that it takes a lot of time for a new device to be discovered. Very often it means that the user has already left the area [11]. This overall makes Bluetooth trilateration impractical, however more coarse room-level positioning can be done with other methods.

Currently it is impossible to achieve accuracy below one meter [12] using RF-based technologies present in mobile phones [7, 8, 13]. Time-of-arrival does offer robust performance [11], however for RF this requires specialised equipment, which is why less direct approaches using signal strength and bit error rate have to be used. Sound, being significantly slower than RF, is easily localised to a few centimetres (due to longer time of arrival). Borriello et al. [14] showed that it is possible to emit $21 \mathrm{KHz}$ (just above the human hearing range) signal from a mobile phone speaker and successfully receive with a conventional microphone. In a separate study Peng et al. [15] showed that it is possible to utilize sound in order to measure the distance between two mobile phones using time-of-arrival. These two principles are combined in our method that involves trilateration of an inaudible ultrasound signal using a static microphone array. Sound positioning is discussed in greater detail in the next section.

The comparison of positioning methods available for most smartphones is given in Table 1.

TABLE I.

COMPARISON OF POSITIONING METHODS FOR SMARTPHONES.

\begin{tabular}{|c|c|c|c|c|}
\hline & $\begin{array}{c}\text { works } \\
\text { indoor }\end{array}$ & accuracy & $\begin{array}{c}\text { infrastructure } \\
\text { cost }\end{array}$ & reliability \\
\hline GPS & no & poor (n/a) & none & good \\
\hline GSM & yes & average & none & good \\
\hline Wi-Fi & yes & good & none/average & good \\
\hline Bluetooth & yes & good & average & poor \\
\hline Sound & yes & excellent & $\begin{array}{c}\text { average/ } \\
\text { expensive }\end{array}$ & good \\
\hline $\begin{array}{c}\text { Computer } \\
\text { Vision }\end{array}$ & yes & excellent & none-average & poor \\
\hline
\end{tabular}

\section{B. Sound Positioning}

Sound is a mechanical wave which travels at speeds much lower than the speed of light. In dry air at a temperature of $25^{\circ} \mathrm{C}$ the speed of sound is only $346 \mathrm{~m} / \mathrm{s}$. At such propagation speeds, one sample of a standard $44.1 \mathrm{KHz}$ stream (44100 cycles/second) accounts for $0.8 \mathrm{~cm}[7,16]$. In other words a signal will travel only 0.8 centimeters in the duration of the smallest time grain. Technically it is possible to work with sound even at $384 \mathrm{KHz}$, which can give much finer accuracy. Unfortunately, an audio recording does not have a reference point for when the signal was sent, it has to be collected therefore from the sender. If the sender and receiver have clock skew/drift between each other, this will result in synchronization uncertainty. One more uncertainty results from possible misalignment between the time a command to emit sound was issued and the actual emission time. Finally, receiving uncertainty occurs as a possible delay in the signal being recognised.

Peng et al. showed that all of the above uncertainties can be eliminated when estimating distance between two devices [8]. Their "BeepBeep" ranging procedure involves two mobile devices starting to record sound before emitting short sound signals one after another. This way each recording has two reference points. Device A has a recording of the signal emitted by device A reaching the microphone on device A, and later of the signal emitted by device B reaching device A. Device B has a recording of the signal from device A reaching device $B$ followed by the signal from Device B reaching device $B$. The span between the two signals on device A is longer than on device B since device A was the first one to emit sound. When the second span is subtracted 
from the first span the result is equal to twice the time it takes sound to travel between the two devices. (Figure 1)

"BeepBeep" has presented itself very well in open environments, but unfortunately showed poor accuracy indoors at distances longer than 5 meters. Most likely this was caused by the multipath effect. The experiments were done in a small room with one or the other device close to a wall, which interprets a signal that bounced off a wall to be of comparable strength to one that arrived via the shortest path.

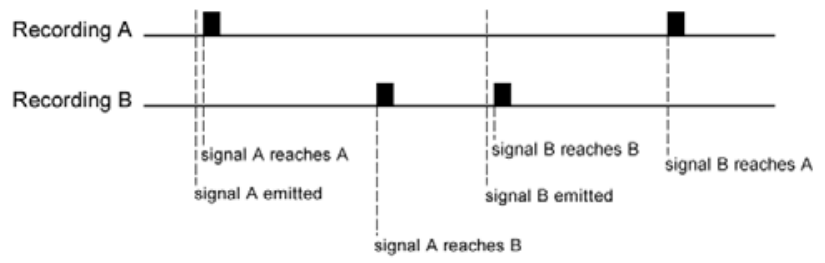

Figure 1. BeepBeep signal exchange. The two horizontal lines represent recordings on each of the devices. Black boxes are actual sound signals that were recorded. The dashed lines represent events. Time interval between the two boxes on recording A minus time interval between the two boxes on recording $\mathrm{B}$ equals $2 \mathrm{x}$ the time it takes for the signal to travel between the two devices.

"BeepBeep" presents a very good idea that overcomes several problems common to acoustic ranging systems, but unfortunately the procedure is not very suitable for trilaterartion. To provide the necessary measurements, there has to be at least three or four visible beacons which allows for measuring distance to them simultaneously either by listening to sound signals emitted by the mobile device or simultaneously emitting sound. The first approach seems to be intuitively favourable. Although it does not really eliminate any synchronization problems, many difficulties can be avoided by listening to just one signal at multiple locations. First of all, there is no need to distinguish between several different signals that arrive either simultaneously or very close to each other. Secondly, the computational load of trilateration will be on the server connected to the microphones, rather than the mobile device.

The effective range of transmitting beacons greatly depends on the volume of the signal and the direction of the speaker. Traditionally, a spherical model is used for sound propagation. However, it has also been observed that ultrasound fading follows a water-drop shaped model as in Figure 2, which should be true for sound at higher audible frequencies as well $[7,8]$. Another thing to take into account is the fact that sound at higher frequencies can be easily blocked by furniture. Most smartphones have both a speaker and a microphone on the same side as the display screen while some also have a louder speaker on the opposite side. Regardless if the phone emits or listens for signals, beacons placed on the ceiling will have a direct line of sight with the phone's speaker/microphone while the user is using the phone. For small rooms it should be enough therefore to place a beacon at the top of every corner of the room. Unfortunately the water-drop model suggests that if a room is significantly larger, the angle between a speaker and a microphone will be too great and the signal will fade too much, in which case a number of beacons will have to be placed on the ceiling to form a grid. We suspect that placing microphones flat against walls/ceiling should effectively counter the multipath effect, which speaks in favour of a mobile phone as the signal source.

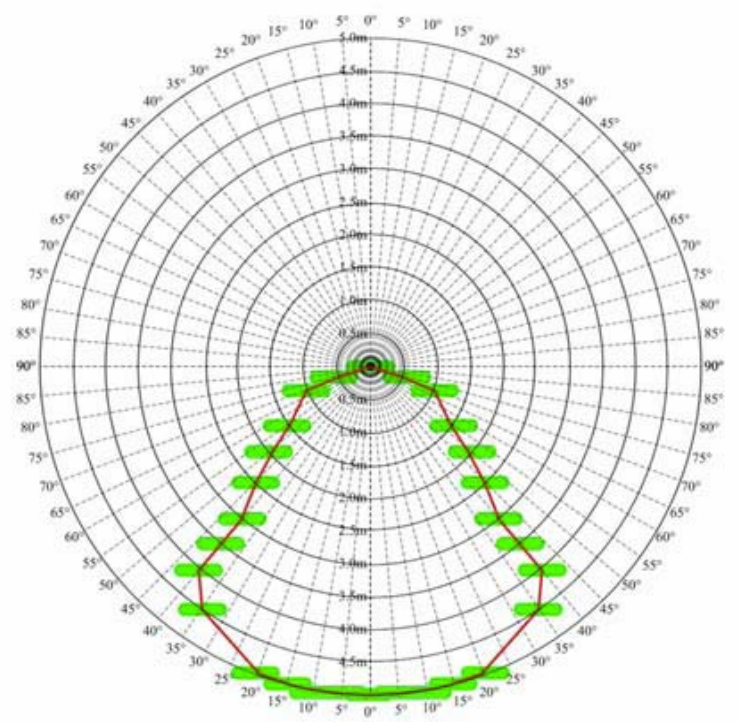

Figure 2. Directional model sound transmission, adapted from Hsiao C.[17].

It is evident from examples given above that the mobile device needs to communicate with the infrastructure somehow, first to communicate the intention to estimate position and secondly to exchange measurement results. It appears impossible to reliably transfer data with conventional speakers and microphones. According to research, the signal to noise ratio even at a range as short as 1 meter is too high to correctly decode more than $95 \%$ of the packets [7]. Wi-Fi communication is a more reliable alternative. As a result the sound signal can be of any length, shape and frequency as long as it can be reliably detected. It has been observed that the first few milliseconds of a sample playback come with a very large distortion which at certain frequencies appear to be a loud unpleasant click [7, 18]. It is therefore recommended to linearly increase the amplitude of the signal. Regrettably, this may introduce some uncertainty to where the beginning of the signal is - an otherwise perfect candidate for a reference point. The end of the signal is a bad choice because it is likely to merge with an echo coming by an alternative path. The multipath effect is also the reason why it is not efficient to determine the middle of the signal and use that as a reference. The best solution appears to be a signal that linearly increases in amplitude and immediately decreases. This will form a "peak" that the receiver will try to detect. Finally the sound frequency presents a choice between efficiency and usability. It has been suggested that anything above $8 \mathrm{kHz}$ attenuates too quickly. On the other hand it appears desirable to use a frequency that is inaudible to the human ear. Frequencies above $20 \mathrm{KHz}$ (ultrasound) generally cannot be picked up by human ear. While these frequencies reduce the effective range of our system, this is offset by a noiseless positioning system placing more importance on user experience. If necessary, this would justify an increase in the number of necessary beacons. Also higher frequencies are easily stopped by obstacles, while 
lower frequencies can even penetrate walls. If taken into account when designing the system either could be used to an advantage.

There are two examples of indoor positioning systems that successfully utilise ultrasound signals: the Bat and the Cricket . These two systems are very similar as both require a dense grid of sensors on the ceiling. Both the beacons and positioning devices are specialised hardware, designed to operate in the ultrasound range. In case of the Bat transmitter, the mobile device transmits a short ultrasound pulse and the time-of-flight from the transmitter to receivers mounted at known positions is measured. Cricket on the other hand uses a combination of radio signals and ultrasound. Beacons periodically transmit "advertisements" on a radio-frequency channel and send an ultrasonic pulse at the same time. Once the locationing device detects an advertisement, it listens for the corresponding ultrasonic pulse. Once the pulse is received, it is possible to calculate the distance by comparing the arrival time of radio and ultrasound signals. Both systems have accuracy of about $3 \mathrm{~cm}$ with the Cricket being slightly more accurate. Also both systems have proved to be highly scalable being able to operate on multiple devices and over large areas. For example the Bat system was installed throughout a threefloor 10,000 square foot office building, possible with 750 beacons, and continuously tracks 200 mobile devices [12]. Overall the ultrasound approach is a perfect solution for indoor positioning in terms of accuracy and we are very interested in finding a way to implement it on conventional off-the-shelf hardware, as that would potentially make it very cheap and accessible, with both microphones and speakers being mass produced and widely available.

It was shown by Borriello et al. that $21 \mathrm{KHz}$ signals can be successfully emitted and received with conventional desktop speakers and microphones (on a HP iPAQ 3870 PDA and a Dell Inspiron 8200 laptop) [14]. The signal was also successfully detected $100 \%$ of the time within a range of 10 meters. This was done using three instances of the Goertzel algorithm: one in the $21 \mathrm{KHz}$ frequency and the other two in adjacent frequencies above and below. The first instance was checked against the other two in order to distinguish the signal from background noise. In order to check how well the detection system copes with common environmental noise three separate tests were performed. One involved a number of people having a conversation, the second involved playing a variety of music recorded in two different formats (mp3 and ogg), and the final test was leaving the system running in an office environment for two consecutive days. During the three tests the detection algorithm did not detect any signals. This is a very encouraging finding, because it means that it may be possible keep working with "raw" sound without introducing complicated filters to check for false positives. The only source of false signals remains the multipath effect, which we hope can be countered with correct placement of microphones and some adjustments in detection algorithms like those proposed in [15].

At the moment ultrasound positioning is the most accurate solution for indoor use. It easily passes the onemeter threshold and comes very close to the one centimetre threshold. So far it has been done with the help of custom hardware, but we see no reason why it could not be done using conventional speakers and microphones.

\section{PROPOSED SOLUTION}

After having reviewed positioning methods available on most modern smartphones, ultrasound trilateration was recognized as a suitable method to deliver fine-grained indoor positioning for the following reasons:

1. Among positioning methods reviewed, only sound positioning can potentially offer consistent sub-meter accuracy. There are good reasons to aim for higher accuracy of estimated position and orientation. To begin with, everything indoors happens on a smaller scale. Corridors are narrower than streets and room entrances are smaller than shop fronts. An indoor LBS is very easy to expand in terms of functionality once all the infrastructure and spatial data is there, so if there is no need for submeter accuracy initially, lack of it should not be a limiting factor for expansion. The requirements for accuracy can be different depending on the task. For example a virtual tour guide with spatial querying will require as fine accuracy as possible at least below one meter, because the deviation will increase as the distance to the object increases. While privacy is a good reason to limit maximum positioning accuracy for pervasive technologies such as GPS, GSM and possibly Wi-Fi, it should not be of concern for sound positioning as it cannot be used to determine position outside the areas equipped with the infrastructure.

2. Ultrasound trilateration is sufficient on its own and will not benefit much from merging with other positioning methods. Among GPS solutions only pseudolites work indoors, but they are currently not compatible with mobile phones. GSM provides no benefit, being less accurate. Some simple form of Wi-Fi positioning may be used to track the user between locations for extra reliability considering a Wi-Fi connection will be needed anyway to send requests and content, however this is not a major issue. Bluetooth performs rather poorly with moving targets. Finally computer vision is a very promising solution on its own, but there is little benefit from combining it with sound trilateration. While computer vision can be very accurate, it will consume a lot of computational resources; require a lot of development and tweaking while at the same time being dependant on how the user operates the phone.

3. Ability to use ultrasound, which is inaudible to human ears, is an important attribute of a system that uses sound waves. If a sound signal used for trilateration was within the hearing range, it would appear sharp, loud and overall unpleasant to human ear. This is because a signal needs to be as distinct as possible in order to cover long distances, resist reverberation and clearly identify time-of-arrival. The concept is very similar to how fiduciary markers in computer vision must be very vivid to allow accurate readings unless the system uses infrared, which is invisible to human eyes.

4. Sound presents an effective way of using trilateration with conventional mobile phone hardware. Because under the same temperature conditions sound travels through air at a constant relatively slow speed, it is possible to accurately deduce distance from time-of-arrival even at an average sample rate. In contrast, electromagnetic waves travel at the speed of light, so Wi-Fi, Bluetooth and GSM 
trilateration has to rely on signal strength, which is a much less reliable parameter.

5. Ultrasound positioning is compatible with many mobile interfaces. Because ultrasound positioning will work regardless of how the user holds the device, it is not restricted to a couple of interfaces such as is the case with computer vision. At the same time high accuracy of positioning means interfaces such as directional querying can be implemented. Finally ultrasound should not disrupt audio interfaces.

The proposed approach is to generate a simple sine tone ultrasound signal using inbuilt mobile phone speakers. The signal is then received by up to four matched DPA microphones, each located in one corner of the test laboratory, and processed using a Pro Tools HD system. Live audio streams from the four microphones are then analyzed in real time by DSP filters tuned to specific ultrasound frequencies. The arrival time at each microphone is then used to calculate the position of the signal source using trilateration. The derived position can then be combined with accelerometer (pitch and roll) and magnetometer (yaw) readings (which are now standard on many smartphones) in order to obtain the position and orientation of the device. This combination of position and azimuth can then used for directional querying of points of interest (POI) within the environment. Wi-Fi connection can be used to inform the server of the client's intention to send the tone, tone's timestamp, client's identity plus any information necessary for whichever LBS uses this positioning service.

\section{EXPERIMENTAL DESIGN}

In order to test the limitations of generating ultrasound signal on mobile devices experiments were done with four smartphones: HTC G1, HTC Hero, Apple iPhone 3GS and Nokia 6210 Navigator.

First of all it was necessary to test the microphone which would be used to detect the signals. There are very few microphones that officially support frequencies up to 22 $\mathrm{KHz}$. A majority of professional microphones officially cover $20 \mathrm{~Hz}$ to $20 \mathrm{KHz}$, with cheaper models sometimes stopping at $17 \mathrm{KHz}$. This is only a precaution and microphones are known to capture frequencies above the upper limit given in the specifications. Since with microphones the specifications cannot be relied on, it is necessary to confirm that the chosen microphone can detect signals in the entire rage, before each of the mobile phones can be tested. Neumann U 87 Ai microphone was successfully tested by playing one of the sound files, described later in this section, through Beyerdynamic DT 150 earphones at high volume. The specifications for these earphones state they can produce frequencies up to $30 \mathrm{KHz}$.

In order to eliminate any incidental sounds, the experiments were done in a soundproof recording booth. The recording was done using one Neumann U 87 Ai microphone and Pro Tools software.

Initially one $44.1 \mathrm{KHz}$ "WAV" sound file was generated using WaveLab software. This file starts with 10 seconds of silence in order to allow enough time to place the phone in front of the microphone, close the door and start recording. These ten seconds are followed by 11 one second long signals ranging from 17 to $22 \mathrm{KHz}$ with a half $\mathrm{KHz}$ step.
There is a gap of one second between each signal. A spectrogram of this file can be seen on Figure 3.

During the early stages of the experiment it was observed that mobile phones generate a lot of noise in the lower frequencies when playing some or all of the given signals at maximum volume. This effect fades or disappears differently on different devices when volume is decreased. To reflect on that the testing procedure was modified. First of all four more modifications of the sound file were generated where volume is decreased by 20, 40, 60 and 80 percent. Secondly each of the five files was played at maximum volume on the device as well as one and two steps lower from maximum. This resulted in 15 separate recordings per device or 60 altogether. A spectrogram was generated for each of the 60 recordings using Praat software for further analyzis.

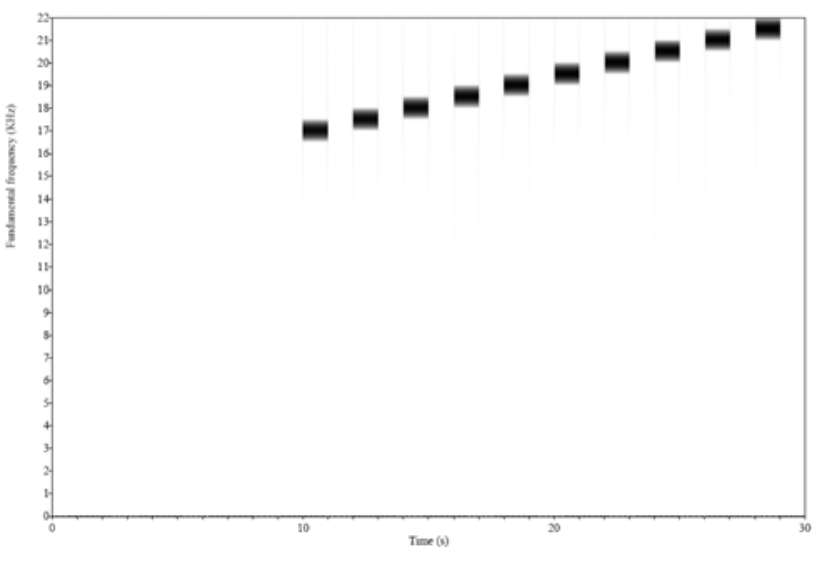

Figure 3. A spectrogram of the file played back by the smartphones. $\mathrm{X}$ axis depict time and $\mathrm{Y}$ axis depict Frequency. Chromatic value shows energy.

\section{DISCUSSION}

Based on the spectrograms generated during the experiment the following observations were made:

1. All tested devices are able to generate all of the given frequencies under the condition that the volume is not too high. In other words there was always energy in the part of the histogram corresponding to the signal. Also for every device it is possible to find a volume setting at which the spectrogram looks almost the same as the spectrogram of the original file. For example with G1 the settings will be file volume $80 \%$, device volume maximum - 2 .(Figure 4)

2. If the volume is set too high, mobile phones will generate a lot of noise in a wide range of frequencies in the audible range when trying to generate one of the signals. For iPhone this happens only with 21.5 and $22 \mathrm{KHz}$, but for Hero and Navigator this happens at all tested frequencies. (See Figures 5 and 6.) Only HTC G1 appeared to be almost completely immune to this problem. As the volume is decreased, this problem fades, and at some point disappears. For example with HTC Hero this happens at around $80 \%$ file volume at maximum device volume. With iPhone noise at 21.5 and $22 \mathrm{KHz}$ disappears completely around $20 \%$ file volume and device volume maximum - 2. 


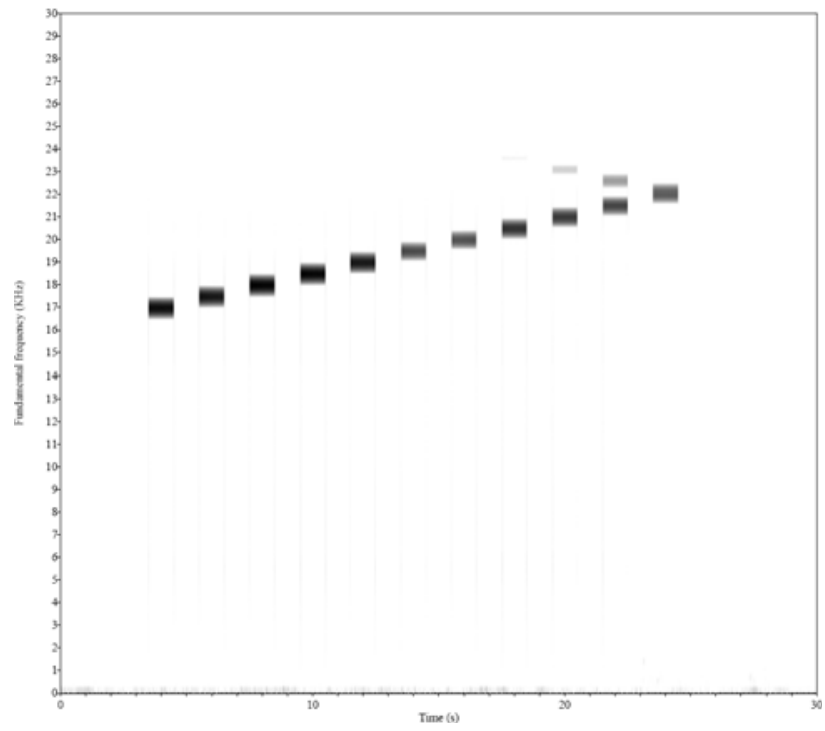

Figure 4. Spectrogram for HTC G1 at file volume 80, device volume maximum - 2

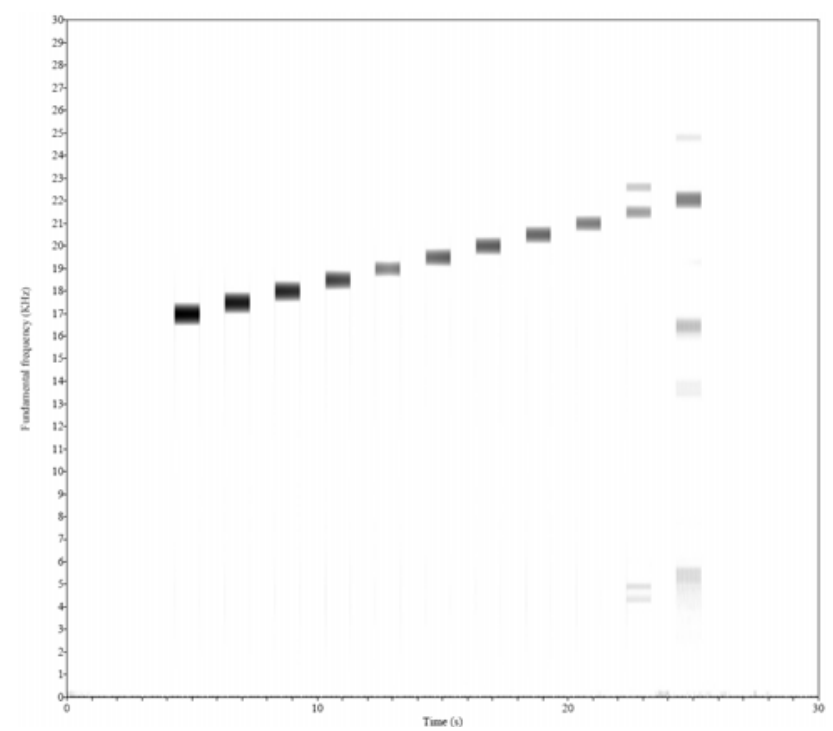

Figure 5. Spectrogram for iPhone at file volume $60 \%$, device volume maximum

3. Volume settings of the device have a major impact on the appearance of noise. This was particularly vivid with Nokia Navigator, where it was impossible to avoid noise even with $20 \%$ file volume. Noise almost completely disappeared when the device was set to maximum - 2 even with $100 \%$ file volume. With other devices it was only observed that noise can be almost completely eliminated by setting the device volume only one or two steps lower than maximum. Reducing volume in the file seemed to have less impact. (See Figure 7 and 8 for comparison)

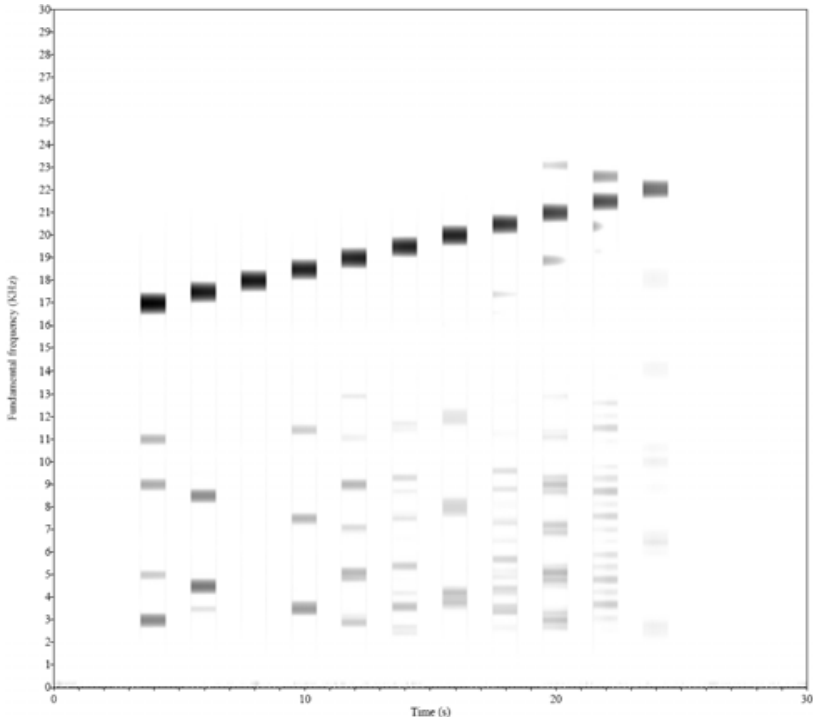

Figure 6. Spectrogram for HTC Hero at file volume $100 \%$, device volume maximum

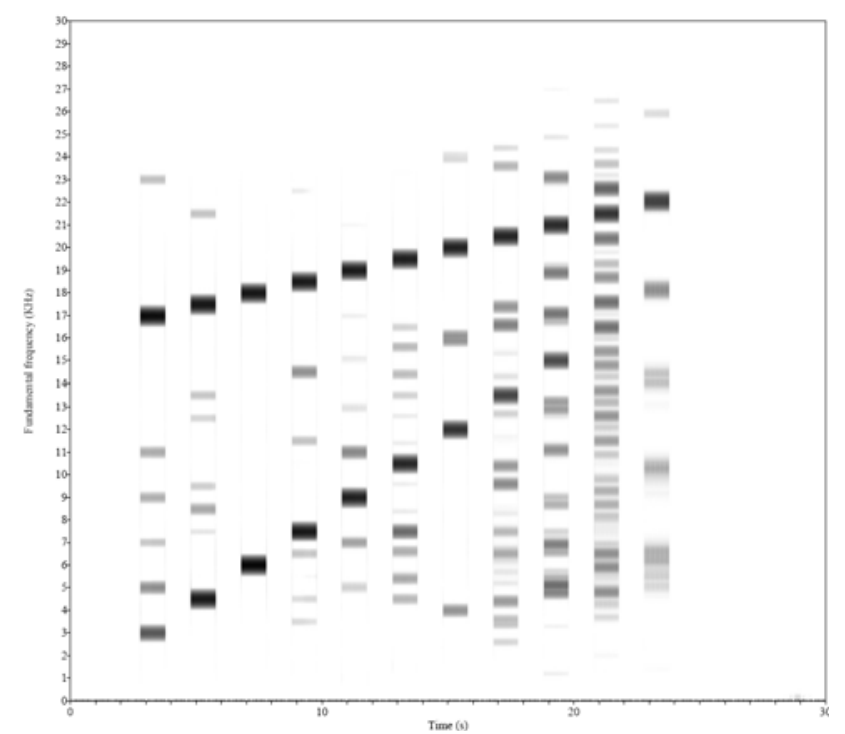

Figure 7. Spectrogram for Nokia Navigator at file volume 20\%, device volume maximum. There is a lot of noise even despite very low volume of the signal in the file.

4. In a majority of recordings there can be observed a particular pattern of artefacts which are a few $\mathrm{KHz}$ higher than the real signals. Sometimes they are almost as powerful the real signal, but very often are hardly visible. A very vivid example can be seen on Figure 8, but for other phones the effect is close to Figure 4. This is probably caused by either resonance in speaker diaphragm or operational errors in Digital Signal Processing (DSP) hardware. This trend may impact scalability of the positioning system. For example as can be seen on Figure 4 , the system wouldn't be able to tell whether the original signal was 21.5 or $22.5 \mathrm{KHz}$. If two different devices used these different frequencies to uniquely identify themselves, the system would fail to tell whether the two signals are an original and a phantom or two simultaneous signals from the two devices. 


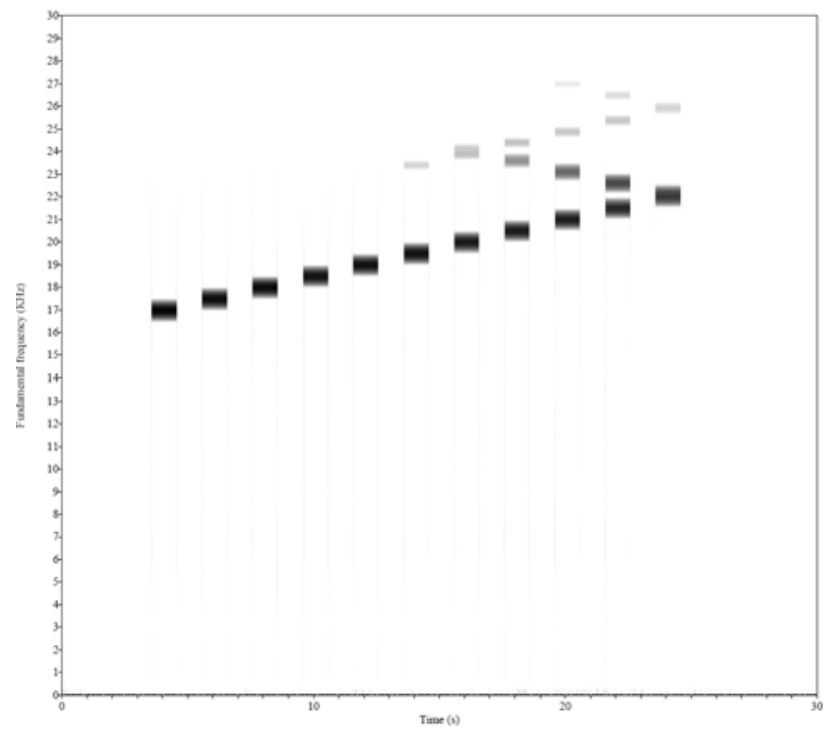

Figure 8. Spectrogram for Nokia Navigator at file volume $100 \%$, device volume maximum - 2. Audible noise abruptly disappears at maximum - 2 settings even though file volume is high.

\section{CONCLUSIONS}

We presented practical limitations of ultrasound generation with mobile phones. With the exception of very high volume settings, all tested mobile phones performed generation of $17-22 \mathrm{KHz}$ signals very well. Some devices performed better than others. HTC G1 didn't generate almost any audible noise even at the highest settings. iPhone showed even less noise at the highest settings with the exception of 21.5 and $22 \mathrm{KHz}$ signals. The other two phones generated a lot of noise at the highest volume settings. The problem with audible noise being generated along with ultrasound was easily avoided by reducing the volume settings on the device. Making the original signal quieter seemed to have less effect or even no effect at all as on Nokia 6210 Navigator. On most devices 20-22 KHz signals were accompanied by noise in the upper frequencies as on Figure 8. Reducing signal volume didn't have almost any effect on them. Although this noise is unavoidable it will not have any impact on usability being inaudible, but should be taken into consideration when scaling up the system to accommodate more devices. From our observations we can conclude that the cause of the noise in the upper frequencies is different from the cause of noise in lower frequencies.

None of the tested devices met any overwhelming obstacles generating inaudible sound frequencies. Combined with what we learned from the literature such as the findings of Peng et al. this makes mobile phone positioning using ultrasound trilateration a promising direction.

\section{FUTURE WORK}

The following questions have to be answered next:

1. What is the maximum distance at which an ultrasound signal emitted by a mobile phone can be reliably detected with a microphone? Sound signals tend to fade with distance and even more so high-frequency signals. At the same time if a signal is very loud it may get distorted by the microphone as well as be audible to some people. Therefore an optimal volume must be found and the maximum distance at which the system can reliably tell it from background noise will be the maximum detection range.

2. Can background noise cause false positives and how this can be countered? There is a possibility that some electric device (e.g. router, network switch, air conditioner, power adapter etc.) in the room produces sound of the same frequency as the signal used by the positioning system and therefore regularly or irregularly causes the system to "detect" a false signal.

3. What kind of ultrasound signal suffers the least from multipath and reverberation under normal room conditions? There are a number of signal properties to experiment with such as volume, frequency, length and shape (e.g. linear increase/decrease of amplitude).

4. What accuracies can ultrasound trilateration offer? First of all it must be found with what accuracy the distance between one speaker and one microphone can be detected. Secondly with what accuracy a mobile device can be located in a 2D plane using an array of microphones. And finally with what accuracy a mobile phone can be located in three dimensions.

5. How can a digital compass be configured to give accurate readings indoors? While this question is not directly linked to positioning, it needs to be answered in order to test how well the proposed method performs with directional querying. Magnetometers are easily distorted by local magnetic fields abundant indoors. They are however expected to exhibit the same deviations in the same locations, so it may be possible to improve accuracy through the process of "weighting", considering accurate position is available.

6. Can the combination of ultrasound positioning and readings from accelerometers and a digital compass be combined to allow for directional querying? This will help evaluate how well the proposed method performs a useful LBS task.

\section{ACKNOWLEDGMENT}

The authors wish to thank the Higher Education Authority (HEA) in Ireland and specifically their Technological Sector Research Strand III: Core Research Strengths Enhancement Programme for funding the work carried out at the Dublin Institute of Technology on the Lok8 project.

\section{REFERENCES}

[1] "Ekahau RTLS Overview.", Ekahau.com, http://www.ekahau.com/products/real-time-locationsystem/overview.html, 21 May, 2009.

[2] M. Meijers, Zlatanova, S., Pfeifer, N., "3D Geo-Information Indoors: Structuring for Evacuation," in First International Workshop on Next Generation 3D City Models, 2005, pp. 11-16.

[3] "Point Inside.", pointinside.com, http://www.pointinside.com, 8 May, 2010

[4] B. Siciliano, Khatib, O., Springer Handbook of Robotics: Springer, 2008.

[5] B. Williams, Klein, G., Reid, I. , "Real-Time SLAM Relocalisation," in Computer Vision, 2007.

[6] D. Wagner, Schmalstieg, D., "First Steps Towards Handheld Augmented Reality," in 7th IEEE International Symposium on Wearable Computers, 2003, pp. 127-136. 
[7] V. Otsason, Varshavsky, A., LaMarca, A., De Lara, E., "Accurate GSM Indoor Localization," in Pervasive and Mobile Computing, 2007.

[8] B. Ferris, Hähnel, D., Fox, D, "Gaussian Processes for Signal Strength-Based Location Estimation," in Robotics Science and Systems 2006.

[9] "Wi-Fi Alliance announces groundbreaking specification to support direct Wi-Fi connections between devices.", wi-fi.org, http://www.wi-fi.org/news_articles.php?f= media_news\&news_id=909, 10 November, 2009.

[10] S. Zhou, Pollard, J., "Position measurement using Bluetooth," Consumer Electronics, IEEE Transactions on, vol. 52, pp. 555-558, May 2006.

[11] K. Kolodziej, Hjelm, J., Local positioning systems : LBS applications and services, 2006.

[12] M. Addlesee, Curwen, R., Hodges, S., Newman, J., Steggles, P., Ward, A., Hopper, A., "Implementing a Sentient Computing System," IEEE Computer, vol. 34, pp. 50-56, August 2001.

[13] J. Hallberg, Nilsson, M., Synnes, K., "Positioning with Bluetooth," in ICT, 2003.

[14] G. Borriello, Liu, Alan., Offer, T., Palistrant, C., Sharp, R., "WALRUS: Wireless Acoustic Location with Room-Level Resolution using Ultrasound," in Mobisys, 2005, pp. 191-203.

[15] C. Peng, Shen, G., Zhang, Y., Li, Y., Tan, K., "BeepBeep: A High Accuracy Acoustic Ranging System using COTS Mobile Devices," in SenSys, 2007.

[16] "Navizon Technical Paper", navizon.com, http://www.navizon.com/Navizon_wifi_gps_and_cell_tower_positio ning.pdf, 11 January, 2009.

[17] C. Hsiao, Huang, P., "Two Practical Considerations of Beacon Deployment for Ultrasound-Based Indoor Localization Systems," in Sensor Networks, Ubiquitous and Trustworthy Computing, 2008, pp. 306-311.

[18] "Skyhook Wireless: How it works", skyhookwireless.com, http://www.skyhookwireless.com/howitworks, 12 May, 2009. 